\title{
GPPS-CH-2020-0089
}

\section{STALL MARGIN IMPROVEMENT IN A LOW-SPEED AXIAL COMPRESSOR ROTOR USING A BLOCKAGE-OPTIMISED SINGLE CIRCUMFERENTIAL CASING GROOVE}

\author{
A. F. Mustaffa \\ Thermo-Fluid Mechanics Research Centre \\ School of Engineering \& Informatics \\ University of Sussex \\ A.F. Mustaffa@sussex.ac.uk \\ Falmer UK BN19QH
}

\author{
V. Kanjirakkad \\ Thermo-Fluid Mechanics Research Centre \\ School of Engineering \& Informatics \\ University of Sussex \\ V. Kanjirakkad@sussex.ac.uk \\ Falmer UK BN19QH
}

\begin{abstract}
The stall margin of tip-critical axial compressors can be improved by using circumferential casing grooves. From previous studies, in the literature, the stall margin improvement due to the casing grooves can be attributed to the reduction of the near casing blockage. The pressure rise across the compressor as the compressor is throttled intensifies the tip leakage flow. This results in a stronger tip leakage vortex that is thought to be the main source of the blockage. In this paper, the near casing blockage due to the tip region aerodynamics in a low-speed axial compressor rotor is numerically studied and quantified using a mass flow-based blockage parameter. The peak blockage location at the last stable operating point for a rotor with smooth casing is found to be at about $10 \%$ of the tip chord aft of the tip leading edge. Based on this information, an optimised single casing groove design that minimises the peak blockage is found using a surrogate-based optimisation approach. The implementation of the optimised groove is shown to produce a stall margin improvement of about $5 \%$.
\end{abstract}

\section{INTRODUCTION}

The near casing flow region of an axial compressor has long been a subject of interest due to its potential link to spike stall inception. For a low-speed axial compressor, the tip region is dominated by low momentum fluid due to casing boundary layer effects and the tip leakage flow (TLF). The low momentum fluid results in blockage effects that intensify as the compressor reaches its stability limit. Furukawa et al. (1999) numerically showed that the rapid rise of the blockage region in a low-speed compressor at near stall conditions can be attributed to the breakdown of the tip-leakage vortex (TLV). The abrupt drop in pressure rise across the rotor is explained by the suction side boundary layer separation due to the interaction with the blockage. In another numerical study in a low-speed environment, Hoying et al. (1999) showed that the TLV trajectory of an isolated rotor becomes more aligned to the circumferential direction as the operating point of the compressor moves closer towards stall. This phenomenon can be explained by the axial momentum balance across the rotor. As the mass flow rate is reduced, the pressure rise across the rotor increases. A further reduction in mass flow rate results in reduced axial flow momentum and a stronger pressure rise. This imbalance pushes the TLV trajectory upstream and causes a 'spill forward' effect.

The 'spill forward' effect can be mitigated by using circumferential casing grooves. An axial momentum analysis by Shabbir and Adamczyk (2005) showed that the stall margin improvement (SMI) by circumferential grooves is due to the radial transport of axial momentum across the grooves. In the axial momentum balance equation, the radial transport of axial momentum at the groove-main flow interface adds a positive term that helps to balance the axial pressure force across the rotor. This delays the 'spill forward' that is thought to be responsible for the stall. Houghton and Day (2009) studied 
the effect of axial variation of the location of a single circumferential groove in a low-speed compressor. They considered a shallow groove and a deep groove for this. For both type of grooves there were two axial locations that produced the best SMI, at $10 \%$ and $50 \%$ axial chord $\left(c_{a x, t}\right)$ aft of the leading edge (LE) over the rotor tip. The casing groove at $0.5 c_{a x, t}$ location provided a higher SMI but also resulted in a higher efficiency penalty with respect to that at the $0.2 c_{a x, t}$ location. The experimental study by Li et al. (2014) also suggest a similar trend to Houghton and Day (2009) in terms of the SMI and groove location. Two peaks of best SMI were found at near LE and near tip mid-chord when a single groove is varied axially across the rotor tip. The above authors explained that the SMI due to the groove were linked to the delay in the upstream movement of the TLF interface. Although both studies above have shown that casing grooves can delay the stall onset, the method used of finding the groove with the highest SMI is based on a trial and error approach. This is because the tests were designed and performed using various groove sizes or locations with no considerations of the internal workings of the groove design tested. It is therefore difficult, without much post-test analysis, to explain why one groove design produces favourable SMI than another groove design. In addition, the outcome of a groove design and its location through such a 'black-box' approach pose open-ended questions regarding the groove's depth, aspect ratio, and its ability to produce the least efficiency penalty.

These questions can only be answered if a design optimisation study is performed. However, a design optimisation study would not address the disadvantage of the 'black-box' approach explained earlier if the SMI itself is chosen as the objective function. This is because the optimiser algorithm has no knowledge about the inner workings of the groove that produces the highest SMI. The aim of this paper, therefore, is to show that a circumferential groove design can be obtained through a 'physics-based optimisation' approach where a blockage parameter is chosen as one of the objective functions. The choice of the blockage parameter is explained in the first part of this paper. Next, the implementation of the blockagebased optimisation approach to obtain a single circumferential groove is presented. Lastly, the SMI and flow features of the optimised casing groove with respect to smooth casing are shown.

\section{COMPUTATIONAL METHOD}

\section{Numerical domain and grid}

The numerical domain and the grid used for this study are shown in Figure 1. The numerical domain is based on an isolated low-speed axial compressor (LSAC) rotor. The rotor blade of the LSAC is obtained through an inverse-design method using MISES (Youngren, H. H., Drela, 2008). The inverse design method is an iterative method used to obtain a desired blade shape from a prescribed blade loading. The aim of the inverse-design method is to obtain a tip-critical compressor rotor that is suitable for passive casing treatment study. Hence, the prescribed blade loading is obtained numerically from an axial compressor that is known to exhibit a spike-stall inception pattern. The 3D rotor blade is generated by radially stacking seven 2D profiles at as many spanwise positions. The hub and casing are parallel to one another resulting in a constant annulus height across the blade. The 3D blade design has a nearly constant exit flow angle (absolute) distribution in the spanwise direction. This is in agreement with the flow angle distribution of the tip critical compressor of which the inverse design is attempted. Aerodynamic design parameters of the LSAC are presented in Table 1.

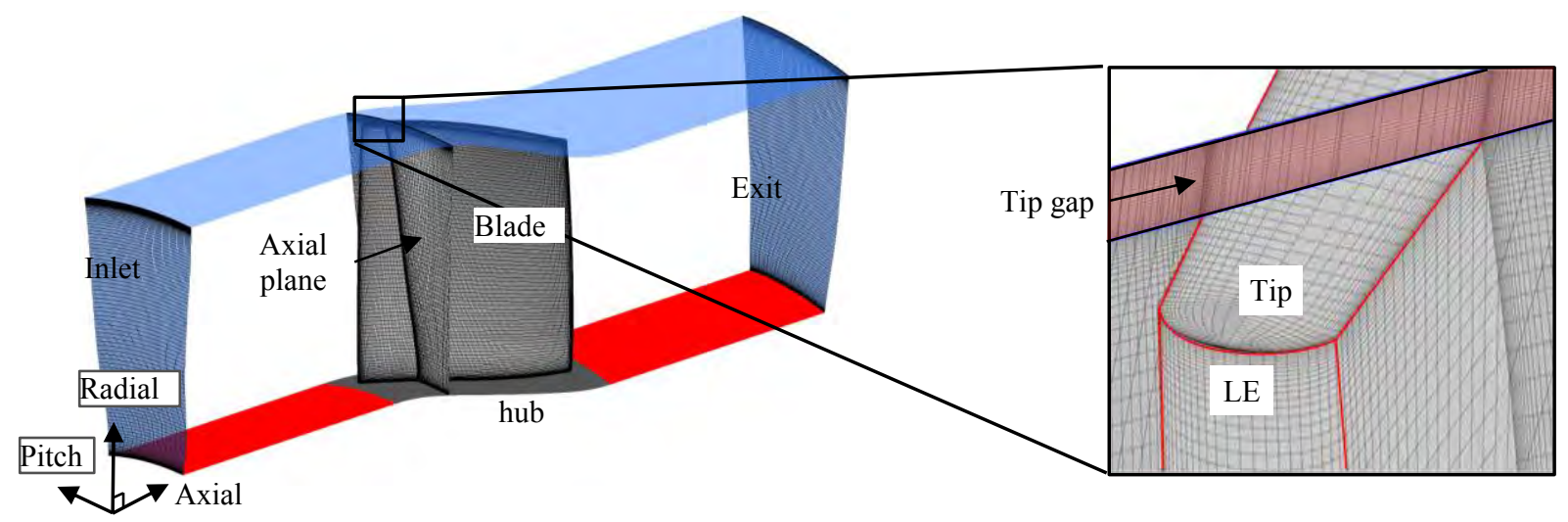

Figure 1 Isometric view of the numerical domain and grid of the LSAC rotor

The single passage numerical domain and unstructured grid are generated automatically using ANSYS Turbogrid 17.1. The grids are generated by setting the target passage grid node count and the boundary layer grid node count near the walls. A grid independence study was conducted by varying the total number of elements, but with a fixed $\mathrm{y}^{+}$value $(\approx 2)$. The change in the converged mass flow rate value and the blade surface static pressure distribution at $98 \%$ span was found to 
be negligible for grid resolutions that are finer. The resulting blade domain has a total grid count of 3.2 million. This is made up of 143 elements in the spanwise direction (of which 30 are in the tip gap region), 72 elements in the circumferential direction and 310 elements in the chordwise direction (of which 147 are within the blade passage). Boundary layer meshing is used in the near wall regions over the blade surfaces (typically 30 layers normal to the blade) and the end wall surfaces.

Table 1 Aerodynamic properties of LSAC

\begin{tabular}{lr}
\hline Stage loading coefficient, $\Psi_{\text {mid }}^{\prime}$ & 0.37 \\
Design flow coefficient, $\phi_{\text {mid }}$ & 0.64 \\
Blade count & 27 \\
RPM & 3000 \\
Hub-to-tip ratio & 0.7 \\
Tip gap height, $\tau[\mathrm{mm}]$ & 1 \\
Tip radius at LE [mm] & 229 \\
True Chord (Tip) [mm] & 68 \\
Reynolds Number (Tip, relative) & $3.2 \times 10^{5}$ \\
\hline
\end{tabular}

\section{Numerical setup and boundary conditions}

The 3D steady RANS equations are solved using ANSYS CFX 17.1 code. The code uses a cell-centred finite volume approach to evaluate the numerical fluxes. Flux values at the cell-boundary are obtained using a high-resolution upwind scheme (ANSYS CFX 17.1 Documentation). The high-resolution upwind scheme is a blended scheme that addresses the shortcomings of the first and second order upwind schemes. The flow is assumed to be fully turbulent and the standard $k-\epsilon$ model is selected for turbulence closure. A scalable wall function is required for resolving the laminar sublayer region (ANSYS CFX 17.1 Documentation). The lessons learned from a previous study, Mustaffa and Kanjirakkad (2019, 2020), of a high-speed rotor with a similar tip loading suggest that the effect of wall bounded viscous phenomena such as separation plays a lesser role in the build-up of the near tip blockage. The above compressor was simulated both at $100 \%$ and at $60 \%$ (where no passage shock was present) speed. This makes the use of the $k-\epsilon$ turbulence model with wall functions justifiable in the current study.

At the inlet, the total pressure and total temperature profiles are prescribed. The steady calculation uses an inlet turbulence intensity of $5 \%$ along with an eddy viscosity ratio of 10 . The total pressure profile used at the inlet boundary corresponds to that of a turbulent boundary layer with a displacement thickness of approximately 5\% of the annulus height. A radial equilibrium exit static pressure condition is imposed at the outlet. Flow within the whole domain is solved in the rotating frame of reference, so all stationary walls are set to be counter-rotating. All walls are treated as smooth and adiabatic. The convergence rate is accelerated using an algebraic multi-grid (AMG) approach built into the solver.

\section{SMOOTH CASING RESULTS AND DISCUSSION}

\section{Performance characteristics}

The performance characteristics of the LSAC for the smooth casing case are shown in Figure 2. The abscissa of Figure 2 is the flow coefficient, $\phi$, which is defined as the average axial velocity at the inlet, $V_{z, 1}$, over the midspan blade speed, $U_{\text {mid }}$. The total-to-static pressure rise coefficient, $\psi_{t s}$, and isentropic efficiency, $\eta$, are calculated using Eq. 1 and 2, respectively. The fluid density, $\rho$, in Eq. 1 and 2 is calculated at the inlet. $P$ and $P_{0}$ are the static and total pressures, respectively and $T_{0}$ is the total temperature. The static pressure in Eq. 1 is its area averaged value at exit whereas the total pressure and total temperatures used in these equations are mass averaged at the appropriate stations.

$$
\begin{gathered}
\psi_{t s}=\frac{P_{2}-P_{01}}{0.5 \rho U_{m i d}^{2}} \\
\eta=\frac{P_{02}-P_{01}}{\rho C_{p}\left(T_{02}-T_{01}\right)}
\end{gathered}
$$

Operating point 1, in Figure 2, corresponds to a hub exit static pressure of $101350 \mathrm{~Pa}$. The characteristic is obtained by gradually increasing the hub static pressure by a value between 50-100 Pa. Operating point 4 and 8 represent the conditions at near design and near stall respectively. The last stable operating point (Operating point 10) is determined iteratively using a 'bisection method' type approach. Here, the size of the hub static pressure increment is updated until any 
further increase of $5 \mathrm{~Pa}$ fails to produce a converged solution. The convergence criteria set for all simulations are as following:

1. The calculations are run for a minimum of 2000 iterations.

2. Coefficient of variation (CV) of the inlet mass flow rate value must not exceed 0.001 for the last 200 iterations. ( $\mathrm{CV}$ is the ratio between the standard deviation to the mean.)

3. The residuals of mass momentum and energy for the last 1000 iterations fall below an acceptable value (typically $10^{-5}$ ) or remain stable below this low value.

The computational methodology used here has been extensively validated in Mustaffa and Kanjirakkad (2019, 2020).
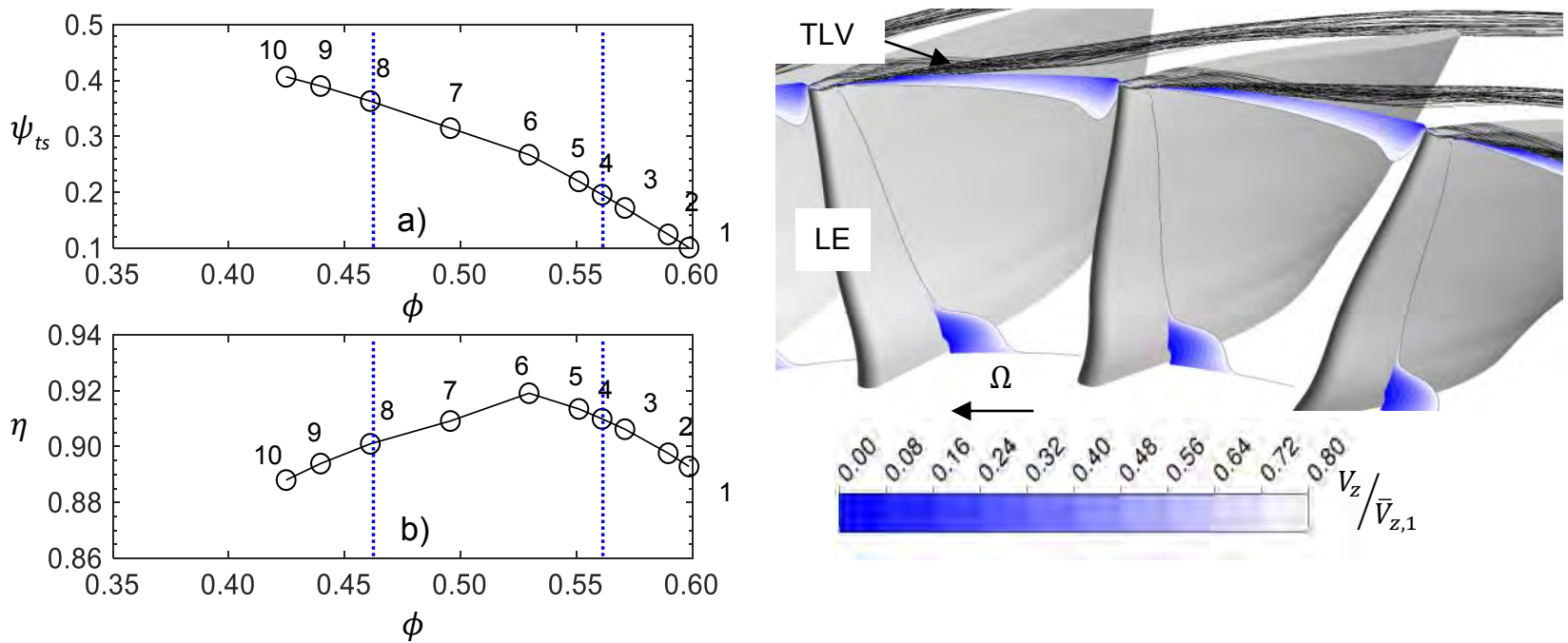

Figure 2 Performance characteristics of the LSAC. a) Total-static pressure rise coefficient and b) Isentropic efficiency

\section{Figure 3 Normalised axial velocity contour plotted for an axial plane located at $0.04 c_{a x, t}$ at operating point 8}

\section{Tip region flow}

As shown in Figure 2a), the stability limit occurs before the $\psi_{t s}$ characteristic reaches its peak (and hence the curve still has a negative slope). A spike-stall behaviour can therefore be inferred based on the findings in Camp and Day (1998). The spike-stall is found to initiate near the tip region when the critical incidence is exceeded. Figure 3 shows the normalised axial velocity contour for an axial plane located at $0.04 c_{a x, t}$ for conditions near stall (operating point 8). The low axial velocity or blockage region (generally seen in Figure 3 as regions where $V_{z} / \bar{V}_{z, 1}<0.8$ ) is predominantly located near the rotor casing. The blockage as seen in the hub region is caused by a separation on the suction side near leading edge and is largely confined radially and circumferentially to the LE suction side hub corner. The blockage region extends much further in the circumferential direction near the casing compared to that near the hub. The findings here further suggest that the LSAC is indeed a tip critical compressor that is suitable for casing treatment.
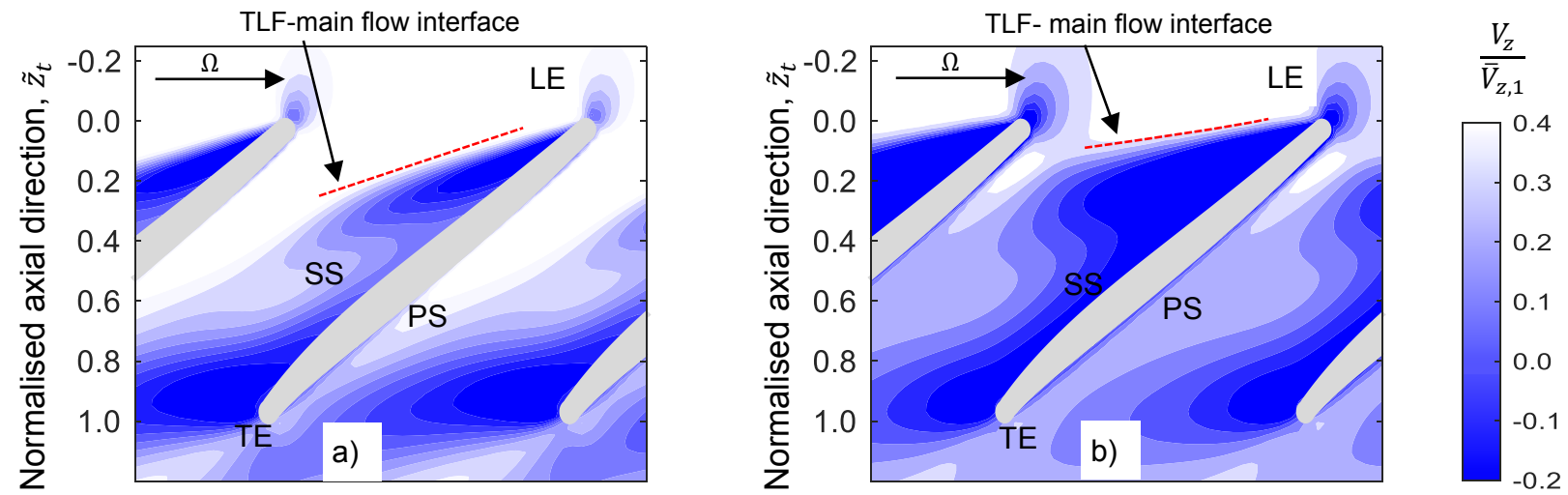

Figure 4 Normalised axial velocity contour inside the tip gap (50\% of tip gap height, $\tau$ ) for a) operating point 4 and b) operating point 8 
The build-up of blockage near the casing as the compressor approach stall can be investigated by plotting the normalised axial velocity contour inside the tip gap for operating point 4 (near design) and 8 (near stall) as shown in Figure 4 . In comparison to operating point 4 , the blockage region due to the TLF at operating point 8 occupies a relatively larger portion of tip gap region within the blade passage. This is expected since the TLF is pressure-driven such that at a lower $\phi$, the blade loading increases (close to the blade tip LE) with increasing $\psi_{t s}$. This results in a stronger TLV and TLF with an increased pitch-wise velocity component. It is interesting to note that there are two regions of increased axial velocity deficit within the passage; one in the forward part of the blade in line with the leading edge tip leakage vortex and the second region close to the blade trailing edge. Although not shown here, these regions are consistent with the local blade (tip) loading distribution.

In addition, at operating point 8 , it can be seen that the interface of the TLF and the incoming flow is more aligned to the circumferential direction compared to operating point 4. This is similar to what has been described in Hoying et al. (1999) where, at near stall conditions, it is found that the upstream movement of the TLV eventually leads to a 'spill forward' effect. The blockage near the blade LE of the suction side (SS) is likely to be caused by a stronger TLV and the increased TLF. The TLV trajectory can be found by extracting the normalised static pressure profiles along several constant pitch lines as shown in Figure 5.
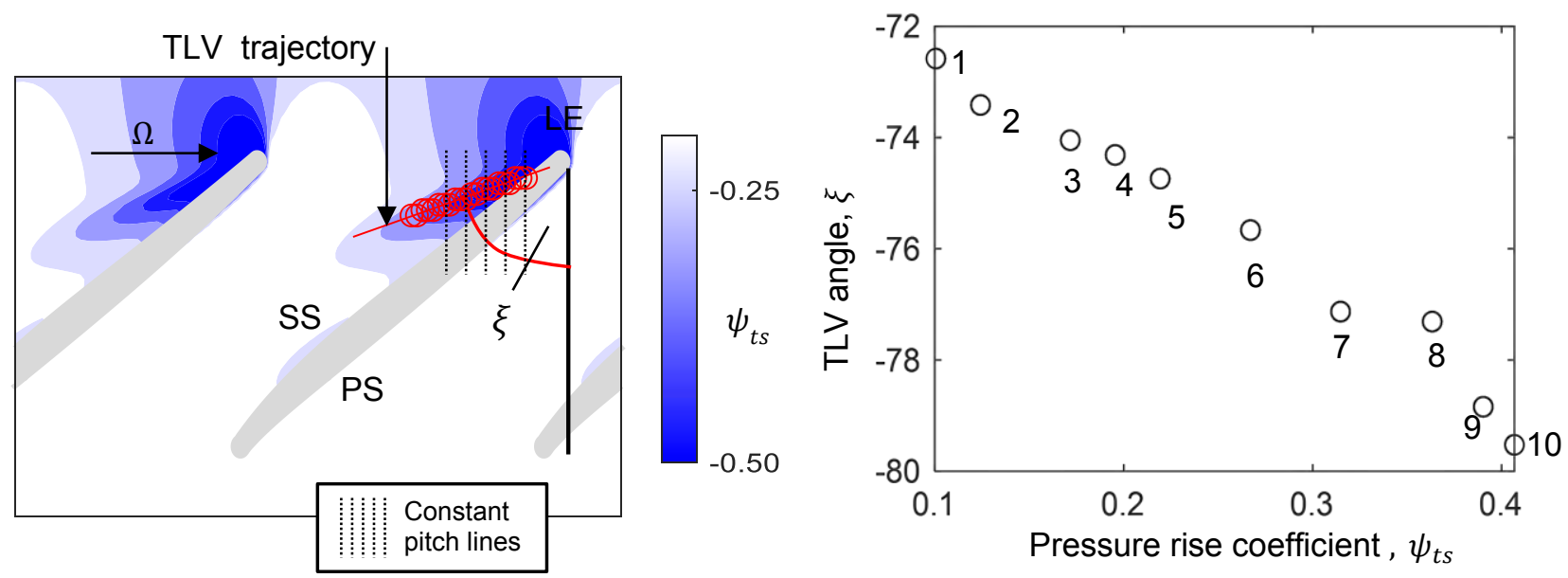
Figure 5 TLV trajectory (red line) extracted from static pressure contour at mid tip gap at operat- ing point 4

\section{Figure 6 TLV angles with respect to the axial di- rection for all operating points}

For each constant pitch line, the axial coordinate of the minimum static pressure is found through interpolation. The trajectory of the TLV can be found through linear fitting. The slope of the TLV trajectories is represented as TLV angle, $\xi$. The angle $\xi$ is measured from the axial direction and is positive if measured in the direction of rotation. This procedure is repeated for all operating points and the TLV angles are plotted as shown in Figure 6. Here the variation of $\xi$ is plotted against the pressure rise coefficient, $\psi_{t s}$, with annotations for the respective operating points as found in Figure $2 \mathrm{a}$ ). As the backpressure increases from operating point 4 to operating point 10 , the change in $\xi$ is about $4^{\circ}$. The increase in $\xi$ and a stronger TLF contributes to the upstream movement of the TLV-main flow interface.

\section{Tip leakage momentum}

As mentioned earlier, the increased loading near the blade tip LE at conditions close to stall, contributes to the increase of the TLF pitch-wise velocity component which, in turn, appears as blockage due to reduction in the axial velocity component. The strength of the TLF that also affects the strength of the TLV can be quantified by calculating the TLF momentum inside the tip gap region as shown in Equation 3.

$$
\mathrm{M}_{\text {tip }}=\frac{\rho V_{\perp}^{2}}{\bar{\rho}_{1} U_{\text {tip }}^{2}}=\frac{V_{\perp}^{2}}{U_{\text {tip }}^{2}} \because \rho \approx \bar{\rho}_{1}
$$

Here, $\mathrm{M}_{\text {tip }}$ is the tip leakage flow momentum normalised by the blade tip momentum where $U_{\text {tip }}$ is the blade tip speed. $V_{\perp}$ is the local velocity normal to the blade camber at the tip. The density, $\rho$ can be omitted considering that at low-speed conditions, the flow is incompressible. The TLF momentum is calculated for operating point 4 and 8 as shown in Figure 7. The shaded region (between the tip LE and 20\% of $c_{a x, t}$ aft of tip LE) represents the area where the increase in $\mathrm{M}_{t i p}$ due to the increased blade tip loading is significant. Within the shaded region it can be seen that $\mathrm{M}_{\text {tip }}$ at near stall conditions 
(operating point 8 ) is about $30 \%$ higher with respect to operating 4 . The increased leakage momentum contributes to reduced axial velocity in the tip region. This coupled with the increase in back pressure at near stall conditions causes the upstream movement of the TLF-main flow interface. Interestingly, as the stall is approached, the tip leakage momentum is seen to decrease in the rear part of the blade $\left(\tilde{z}_{t}>0.5\right)$. This is in agreement with the normalised axial velocity contours presented in Figure 4. The contours suggest that compared to the near design case, closer to stall, the low momentum region near the LE intensifies and moves upstream whereas that near the blade TE suction side becomes less intense. This is due to a reduction in the blade loading in the rear part of the blade. It is thought that the modification of the passage flow by the increased upstream blockage is responsible for this effect.

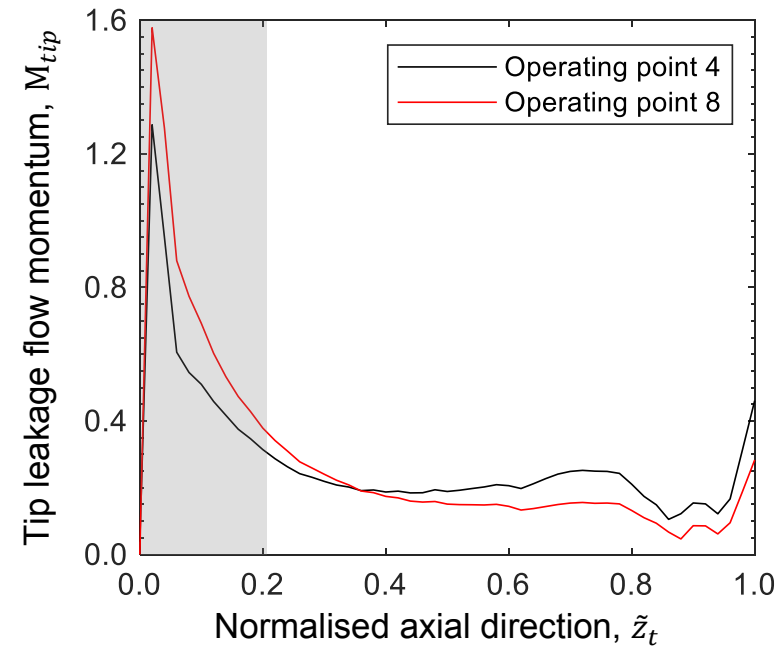

Figure 7 Comparison of normalised tip leakage momentum at operating point 4 and 8

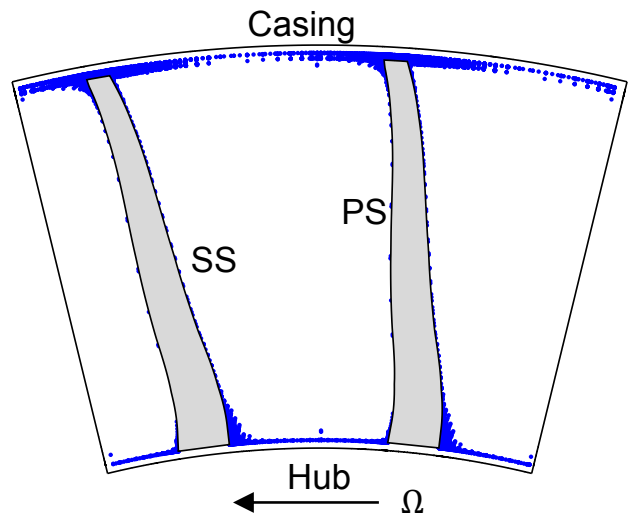

Figure 8 Location of the 'blocked' cells at an axial plane location same as in Figure 3 at operating point 8 (looked from the front).

\section{Quantification of near casing blockage}

The preceding discussion has shown that the upstream movement of the TLF-main flow interface can be linked to the increased blockage (axial velocity deficit) regions at conditions near stall. It is, therefore, thought to be a worthwhile exercise to quantify this blockage to further explore its link to the initiation of stall. The blockage quantification method is based on a mass flow overshoot method introduced by Sakuma et al. (2013) in order to identify and visualise 'blocked' cells in an axial plane. Blocked cells are identified by sorting and summing the mass flow of each grid cell in that plane in a descending order. If cells with negative axial velocity (flow reversal) values exist, the sum of all positive axial velocity will overshoot the inlet mass flow value. The summation is stopped when the summed mass flow reaches the inlet mass flow value. Cells that are yet to be summed are considered as 'blocked' and are assigned a binary blockage index $(\psi)$ of 1 . 'Un-blocked' cells are assigned a $\psi$ value of 0 . The outcome of this method is shown in Figure 8 where the blue dots represents the location of the 'blocked' cells $(\psi=1)$ over an axial plane $0.04 c_{a x, t}$ aft of the blade tip LE. Note that this is the same axial plane as in Figure 3 where the normalised axial velocity contours were presented.

Based on the overshoot method described above, a non- dimensional blockage parameter, $\Psi_{m}$, is introduced to quantify the blockage in that plane. $\Psi_{m}$ is the normalised absolute sum of the 'blocked' cell mass flow rate. The definition of $\Psi_{m}$ is shown in Equation 4.

$$
\Psi_{m}=\frac{\sum_{i=1}^{N}|\psi(i) m(i)|}{\sum_{i=1}^{N} m(i)}
$$

Here, $N$ is the total number of cells in the plane. $\psi(i)$ and $m(i)$ are the blockage index and the mass flow rate respectively that are associated with the $i^{\text {th }}$ cell on this plane

Figure 9 shows the distribution of $\Psi_{m}$ across the rotor blade for all the operating points that are shown in the performance map in Figure 2. The distribution is obtained by extracting data for about 100 axial planes across the blade. Here, the distribution of $\Psi_{m}$ is only evaluated for the outer $20 \%$ span region. This is mainly because the blockage in the tip region is found to be more significant than at the hub region for a tip-critical compressor. The peak blockage located at approximately $0.15 \tilde{z}_{t}$ near design (operating point 4 ) grows rapidly as the compressor is throttled while gradually moving upstream. At the last numerically stable point (operating point 10 ) the peak blockage has grown almost eight times compared to the near design and has also moved upstream, albeit small amount, by approximately $3 \%$ of $c_{a x, t}$. The increased blockage 
is consistent with the TLF momentum increase at conditions near stall as shown in Figure 7. However, the relatively small upstream movement of the location of the peak blockage as the compressor approaches stall, seems to suggest that the peak is likely due to the build-up of blockage originating from the TLV. The TLV trajectory approximation exercise presented in Figure 6 also showed a moderate $4^{\circ}$ change in TLV angle $(\xi)$ from operating point 4 to 10 . The rise in the peak value of the blockage at conditions near stall points to a stronger roll-up of the TLV that is a result of the increase in TLF momentum. This in turn causes an increase in the axial velocity deficit near the LE region. This increase of the axial velocity deficit caused by the TLV therefore results in the upstream movement of the TLF-main flow interface near stall.

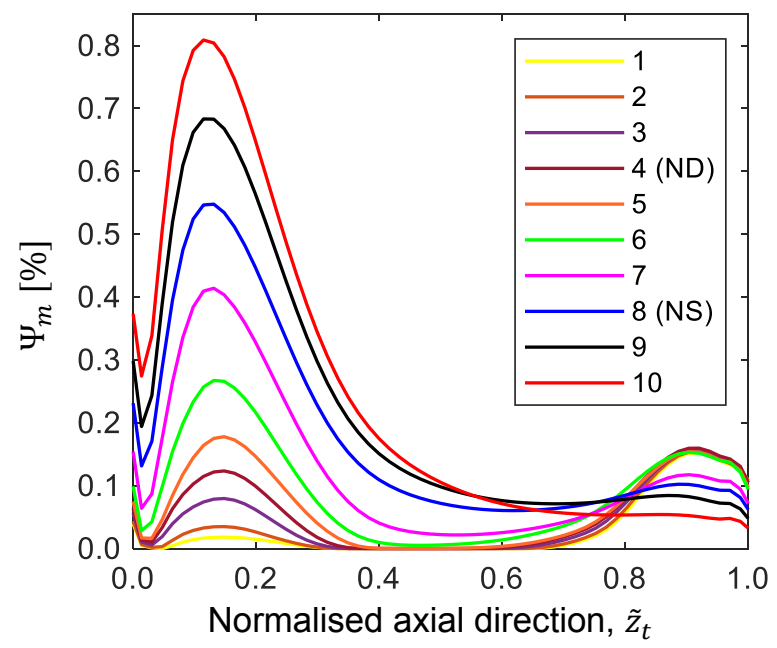

Figure 9 Distribution of $\Psi_{m}$ across the blade for all operating points

Near the TE $\left(\tilde{z}_{t}>0.8\right)$, a small hump in the blockage distribution is visible for all operating points with flow coefficient above that of the NS case. This is consistent with the previous discussion on the appearance of a low momentum region near the TE with reference to Figure 4 and Figure 7. As noted before, at conditions near stall, this low momentum region weakens, which in turn result in the flattening of the hump region as seen for operating points 7 through to 10 .

\section{CIRCUMFERENTIAL GROOVE RESULTS AND DISCUSSION}

The blockage quantification method has established the link between blockage and the inception of rotating stall. Based on this, a design optimisation method to obtain a single circumferential groove is attempted. The objective of the optimisation is to find a single groove design that minimises the peak blockage with the least efficiency penalty. A method that couples a surrogate modelling technique to a multi-objective evolutionary algorithm is employed. The primary aim of using the surrogate model is to reduce the computational cost associated with high fidelity CFD simulations. The surrogate model used is a supervised-learning tree-based algorithm (Breiman, L., 2001) called Random Forest (RF). RF is an ensemble of decision trees that can be used for predicting the response of continuous variables. The RF is constructed from input and output training data. The input training data is generated using a space-filling design sampling technique called Latin Hypercube Sampling (LHS). First, the groove design is parameterised to define the shape of the groove using four variables that are described later in Figure 10a). The shape of the groove is obtained by sampling 100 datasets across a predefined design space boundary. The LHS technique ensures that population of groove shapes generated covers the whole spectrum of the design space. The output data are obtained through CFD simulation of the 100 groove shapes at a single operating condition corresponding to operating point 8 in Figure $2 a$ ) which represents the near-stall condition.. The peak blockage and efficiency of each groove test case is interrogated. The optimisation algorithm implemented is a Multi-Objective Genetic Algorithm (MOGA). The optimiser performs a random search method and outputs a set of groove designs that are Pareto-optimal solutions. The set of groove designs from the Pareto-optimal solution are verified through CFD. The best case is chosen to be the groove design that numerically stalls at the lowest flow coefficient using the previously mentioned convergence criteria. Further details regarding the surrogate-based optimisation technique can be found in Mustaffa and Kanjirakkad $(2019,2020)$ where a similar approach was applied to an isolated transonic rotor.

The optimal single groove solution is obtained as parameters $z^{\prime}, w^{\prime}, H$ and $\alpha$ as shown in Figure 10a). The parameters $z^{\prime}, w^{\prime}, H$ and $\alpha$ are the normalised groove axial position, upper width, height and the upper internal angle, respectively of the groove. All parameters except $\alpha$ are normalised by the blade tip axial chord $\left(c_{a x, t}\right)$. The optimised values for $z^{\prime}, w^{\prime}, H$, $\alpha$ and $c_{a x, t}$ are $0.094,0.094,0.051,2^{\circ}$ and $40 \mathrm{~mm}$, respectively. The domain and the mesh for the optimised groove generated are shown in Figure 10b). The groove domain has 0.2 million grid points and is connected to the blade domain via a GGI grid connection (ANSYS CFX 17.1 Documentation). 


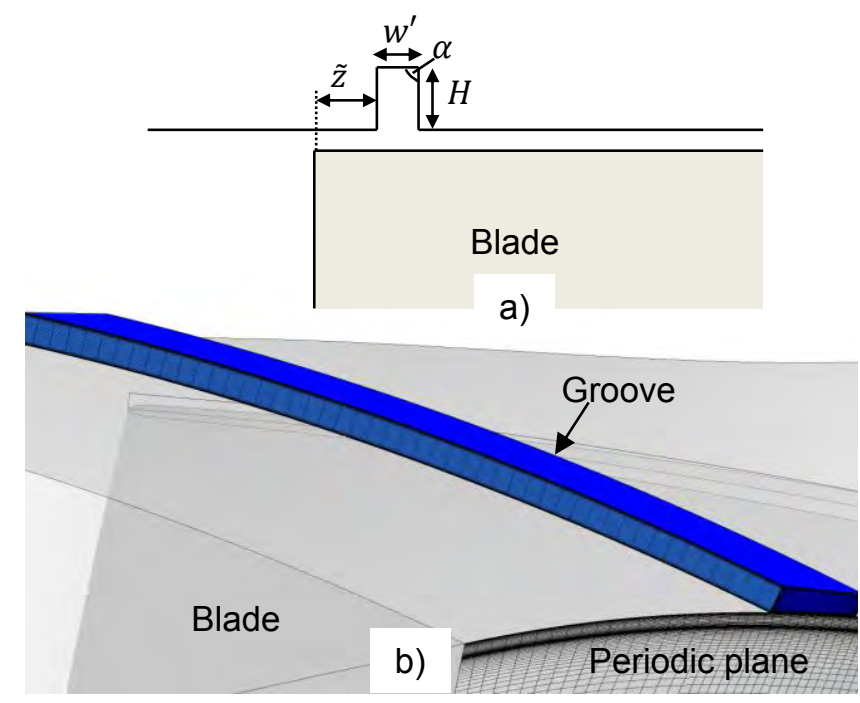

Figure 10 a) Groove parameters and b) optimised groove domain and mesh
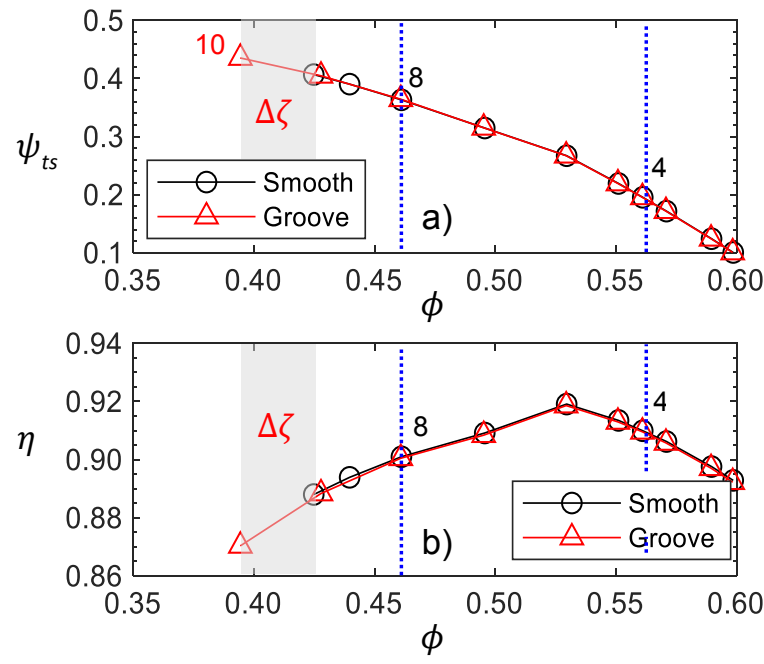

Figure 11 Performance characteristic comparison of the optimised groove and the smooth casing. a) Pressure rise coefficient and b) isentropic efficiency

Figure 11 shows the characteristic of the rotor with the grooved casing in comparison to the one with smooth casing. The SMI $(\Delta \zeta)$ for the optimised grooved casing is calculated using Equation 5.

$$
\Delta \zeta=\zeta_{G C}-\zeta_{S C} ; \quad \zeta=\frac{\left(\phi_{4}-\phi_{10}\right)}{\phi_{4}}
$$

Here, as before, $\phi$ is the flow coefficient which is defined as the average axial velocity at the inlet, $V_{z, 1}$, over the midspan blade speed corresponding to the relevant operating conditions ( $4 \& 10)$. The SMI $(\Delta \zeta)$ thus obtained is $5.4 \%$. The efficiency penalty is calculated to be negligible for each of the operating points simulated as shown by the overlapping efficiency characteristics for the smooth and the grooved cases in Figure 11b). The increase in pressure rise and the dip in efficiency corresponding to the last converged operating point (operating point 10) for the grooved casing are in line with the slopes of the overall trend of the corresponding characteristic curves.

Figure 12 shows the normalised axial velocity contour in the mid-tip-gap for the grooved casing. Here, the TLF-main flow interface (dotted red line) of the grooved casing is shifted further towards the blade suction side than that for the smooth casing shown in Figure 4b). This delays the 'spill forward' effect and hence results in an improved stall margin.

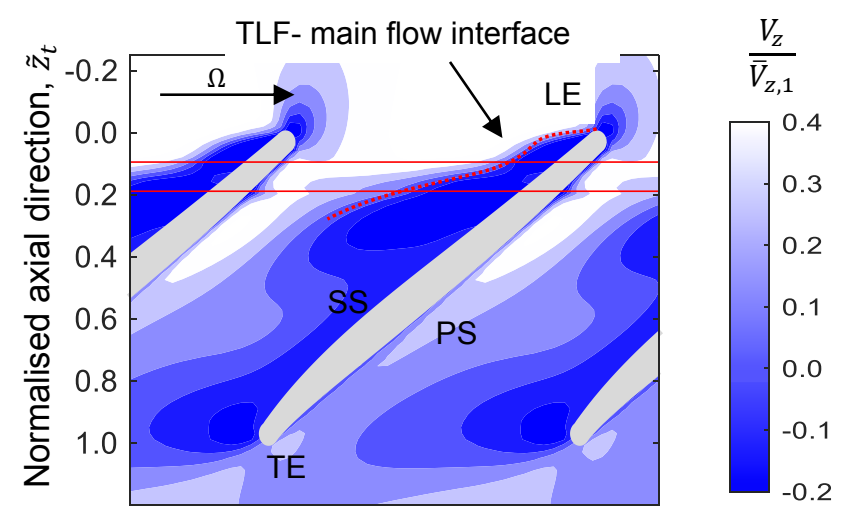

Figure 12 Normalised axial velocity contour inside the tip gap $(50 \%$ of tip gap height, $\tau$ ) for the grooved casing at operating point 8

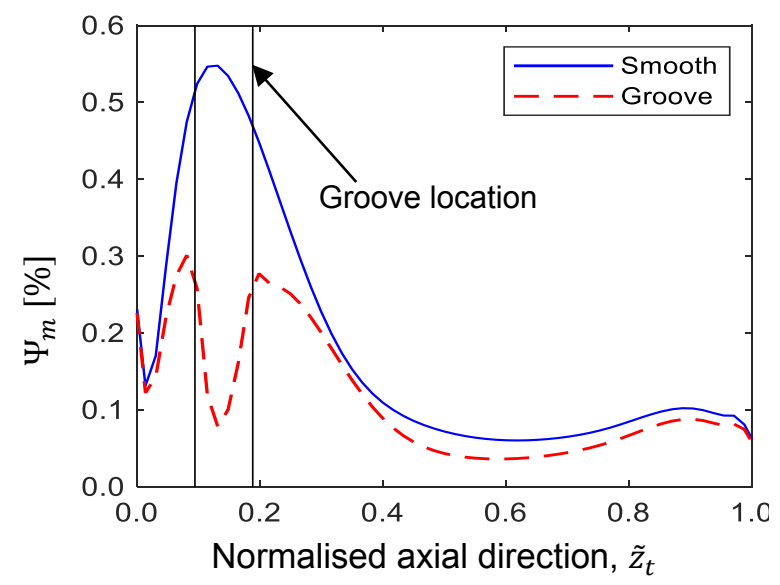

Figure 13 Comparison of the grooved casing blockage parameter, $\Psi_{m}$, at operating point 8 with respect to the smooth casing 
Figure 13 shows the comparison of the non-dimensional blockage parameter, $\Psi_{m}$, at operating point 8 for the smooth and the grooved casing. It is clear that the peak blockage is significantly reduced by the groove as compared to the smooth casing. The presence of the optimised groove therefore reduces the axial velocity deficit in the tip region and hence delays the upstream movement of the TLF interface at conditions near to stall.

Figure 14 shows the non-dimensionalised static pressure contours in the mid tip gap region, with the blade tip profile made visible for clarity. Figure 14a) shows the contours for the smooth casing at near stall operating point (operating point 8.). The tip vortex trajectory obtained by tracking the trough of the static pressure profile is overlaid on top of the contour map. Figure 14b) shows the same contours for the grooved casing. The red line shows the trajectory of the tip leakage vortex developing downstream of the LE of the groove. A dotted line is also shown that represents the trajectory of the smooth casing as before. Clearly, the interaction of the tip vortex with the groove has resulted in a reduced TLV angle. Using the method as described in relation to Figure 5 this reduction in angle is estimated to be $2.7^{\circ}$. This is significant given that TLV angle shift from operating point 4 to operating point 8 is only about $4^{\circ}$ as described earlier. Researchers such as Sakuma et al. (2013) have attributed such a shift in TLV to SMI in a transonic compressor. However the study by Houghton and Day (2009) in a low-speed compressor concluded that TLV modification was not necessary for achieving SMI. But it remains a fact, even in the study by Houghton and Day (2009), that grooves that are positioned downstream of the TLV origin, but closer to the LE do show a modification of the TLV trajectory. This further points to the fact that the positioning of the groove in relation to the peak blockage as implemented in the present study is key to achieving maximum SMI with least efficiency penalty. However, none of the SMI studies as previously mentioned have quantified the blockage inside passage near the tip region and linked it to the improved stall margin that they have reported.

The strategy to quantify the blockage near the casing using a non-dimensional parameter and to use this parameter to obtain a circumferential casing groove that provides improved SMI through an optimisation process has therefore been shown to be successful. This method has already been shown numerically to be useful for improving the stall margin in a transonic compressor rotor by Mustaffa and Kanjirakkad $(2019,2020)$. The flow physics associated with blockage and stall is very different in the transonic environment that is dominated by passage shock and its interaction with the TLV. The outcome of the present study in a low-speed compressor rotor therefore offers the confidence that the physics based optimisation approach adopted here for achieving SMI is a robust one.
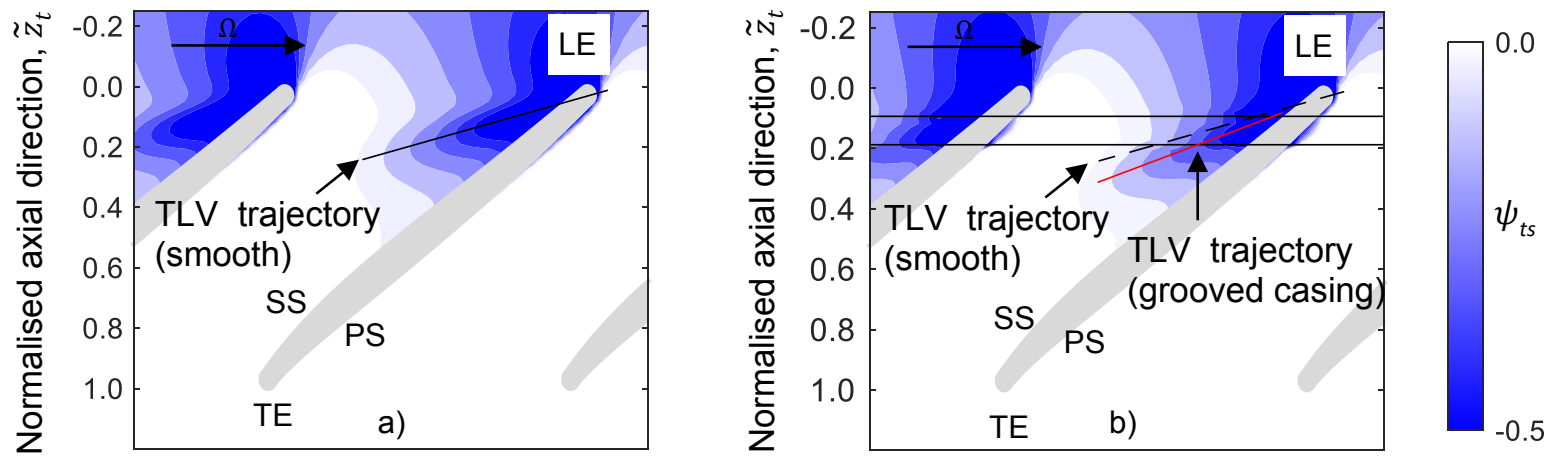

Figure 14 Normalised axial velocity contour inside the tip gap (50\% of tip gap height, $\tau$ ) for a) operating point 4 and b) operating point 8

\section{CONCLUSIONS}

The near casing flow region of a tip-critical low speed axial compressor (LSAC) rotor has been studied numerically. The blockage in the tip region is quantified using a mass flow based non-dimensional parameter. Improvement of stall margin is attempted using an optimised casing groove geometry using a physics based approach that minimises the peak tip blockage as evaluated using the above parameter. The conclusions are summarised as the following:

1. At conditions close to stall, the blade loading increases due to the increased back pressure. This results in a stronger TLV and TLF. By tracking the TLV trajectory (approximated as the locus of static pressure minima) suggests that the TLV angle increases by about $4^{\circ}$ from near design to the near stall operating point. This increase in TLV angle contributes to the upstream movement of the TLF-main flow interface.

2. The strength of the TLF is evaluated by calculating the tip leakage momentum across the tip. At conditions close to stall, it is found that the TLF momentum increases near the LE edge region. The TLF also contributes to the upstream movement of the TLF-main flow interface and the increase of the TLV angle $(\xi)$ as stall is approached. 
3. The near casing blockage region is quantified using a mass flow-based blockage parameter $\left(\Psi_{m}\right)$. It is shown that the location of the peak tip blockage moves upstream from about $0.15 c_{a x, t}$ near design to about $0.10 c_{a x, t}$ near stall. The magnitude of the peak blockage also increases. This is thought to be due to the build-up of low axial momentum as the TLF rolls-up into the TLV. The rise in blade loading causes a stronger TLV roll-up. It is very likely that the TLV is the biggest contributor to the blockage near the casing region.

4. Based on the blockage analysis performed on the smooth casing, a design optimisation is attempted to obtain a single circumferential groove that reduces the peak blockage with least efficiency penalty. The optimised single groove results in a SMI of 5.4\%. The SMI by the groove is shown to be a result of the reduction of the peak blockage magnitude and a shift in the TLV angle. Both factors result in delaying the upstream movement of the TLF-main flow interface.

5. The identification of the location and the minimisation of the peak of the tip blockage by appropriately positioning the groove is therefore recommended as a possible route for designers to achieve SMI through casing modification.

\section{NOMENCLATURE}

$\begin{array}{lll}A & \text { Annulus area } & \Psi_{\text {mid }}^{\prime} \\ H & \text { Groove height } & \Psi_{m} \\ \mathrm{M}_{\text {tip }} & \text { Tip leakage flow momentum } & \Omega \\ P, P_{0} & \text { Static, Total pressure } & \alpha \\ U_{\text {mid }} & \text { Blade speed at mid-span } & \zeta, \Delta \zeta \\ V & \text { Velocity } & \eta \\ \bar{X} & \text { Averaged value of variable } & \xi \\ X_{\text {mid }} & \text { Mid-span value of variable } & \rho \\ X_{\perp} & \text { Normal component to blade chord } & \phi \\ c_{a x, t} & \text { Tip axial chord } & \psi \\ m & \text { Mass flow rate } & \psi_{t s} \\ w^{\prime} & \text { Groove width } & \mathrm{TLV} \\ Z & \text { Axial direction } & \mathrm{TLF} \\ \tilde{z}_{t} & z / c_{a x, t}, \text { Normalised axial direction } & 1,2 \\ z^{\prime} & \text { Groove axial position } & \end{array}$

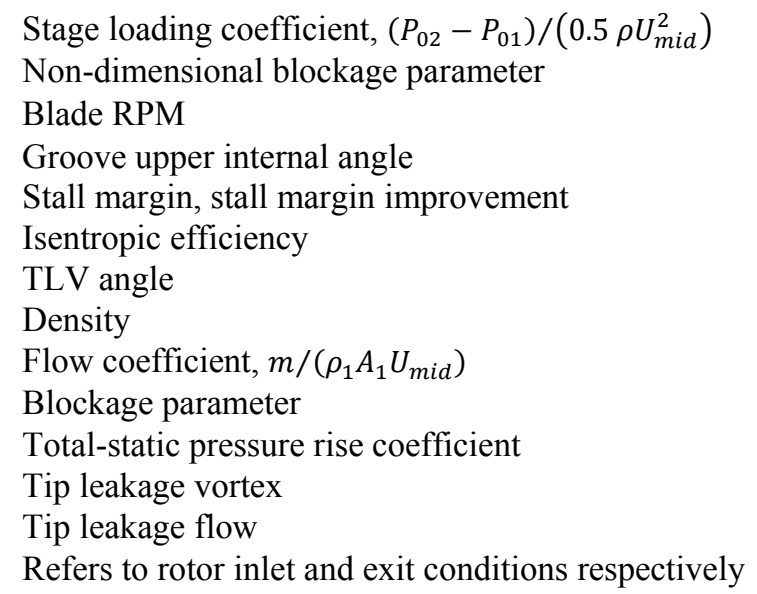

\section{ACKNOWLEDGMENTS}

The first author would like to thank the Ministry of Education, Malaysia and Universiti Sains Malaysia for providing the financial support towards his research studentship at Sussex.

\section{References}

ANSYS CFX 17.1 Documentation. (2016) ANSYS Inc.

Breiman, L. (2001), Random Forest, Machine Learning, Vol. 45 No. 1, pp. 5-32

Camp, T. R. and Day, I. J. (1998) '1997 Best Paper Award-Turbomachinery Committee: A Study of Spike and Modal Stall Phenomena in a Low-Speed Axial Compressor', Journal of Turbomachinery, 120(3), p. 393.

Furukawa, M. et al. (1999) 'The Role of Tip Leakage Vortex Breakdown in Compressor Rotor Aerodynamics', Journal of Turbomachinery, 121(3), p. 469.

Houghton, T. and Day, I. (2009) 'Enhancing the Stability of Subsonic Compressors Using Casing Grooves', Volume 7: Turbomachinery, Parts A and B, 133(April 2011), pp. 39-48.

Hoying, D A et al. (1999) 'Role of Blade Passage Flow Structures in Axial Compressor Rotating Stall Inception', Journal of Turbomachinery. ASME, 121(4), pp. 735-742.

Li, J. et al. (2014) 'Extensive Experimental Study of Circumferential Single Groove in an Axial Flow Compressor', in. Proceedings of the ASME Turbo Expo 2014: Turbine Technical Conference and Exposition. Volume 2D: Turbomachinery. Düsseldorf, Germany. June 16-20, 2014.

Mustaffa, A. F., Kanjirakkad, V. (2019) 'Design Optimisation of Circumferential Casing Groove for Stall Margin Improvement in a Transonic Compressor Rotor', in Proceedings of the 54th 3AF International Conference on Applied Aerodynamics. Paris, France, p. 10.

Mustaffa, Ahmad Fikri and Kanjirakkad, Vasudevan (2020) 'Casing-groove optimisation for stall margin in a transonic compressor rotor', International Journal of Numerical Methods for Heat and Fluid Flow, ISSN 0961-5539 (Accepted)

Sakuma, Y. et al. (2013) 'Numerical Analysis of Flow in a Transonic Compressor With a Single Circumferential Casing Groove: Influence of Groove Location and Depth on Flow Instability', Journal of Turbomachinery, 136(3).

Shabbir, A. and Adamczyk, J. J. (2005) 'Flow Mechanism for Stall Margin Improvement due to Circumferential Casing Grooves on Axial Compressors', Journal of Turbomachinery, 127(4), p. 708.

Youngren, H. H., Drela, M. (2008) 'A User's Guide to MISES 2.63'. 